\title{
Erhöhung der Dimensionalität und Stabilität von Gassensorsystemen als Basis für die erfolgreiche Anwendung von Methoden des maschinel- len Lernens
}

\author{
Caroline Schultealbert, Tobias Baur, Martin Leidinger, Wolfhard Reimringer, Thorsten Conrad \\ $3 S$ GmbH - Sensors, Signal Processing, Systems, Saarbrücken, Deutschland \\ Kontakt: caroline.schultealbert@3S-ing.de
}

\section{Einleitung}

Die Gasmesstechnik gehört zu den herausforderndsten Gebieten der Messtechnik. In der chemischen Analytik wird für jede Messaufgabe die passende Probenvorbereitung (Sorbens, SamplingTechnologie, Transportvorkehrungen) und eine fähige Analysetechnik (z.B. GC-MS, HPLC) ausgewählt. Obwohl Sensorsysteme allgemein als kostengünstig und einfach angesehen werden, gilt für die Gasmesstechnik ähnliches: Sampling, Sensoren, Betriebsart und vor allem Auswertemethodik müssen sorgfältig ausgewählt, vorbereitet und abgestimmt werden. Ein Gesamtsystem ist somit für eine spezifische Anwendung maßgeschneidert. Zum Einsatz kommen dabei oft Methoden des maschinellen Lernens, um die Vielzahl von Sensorsignalen und $\mathrm{Pa}$ rametern erfolgreich zu verarbeiten und in eine relevante Ausgabegröße umzuwandeln.

Ein bekannter und seit mehreren Jahrzehnten verfolgter Ansatz ist der temperaturzyklische Betrieb von Metalloxid-Halbleitergassensoren (MOSSensoren). Der Informationsgehalt eines solchen Sensorsignals ist durch den Zusammenhang von Schichttemperatur und Reaktivität deutlich erhöht [1]. Durch komplexe statistische Kalibrierung und Methoden wie der PLSR (partial least squares regression) oder der Anwendung von neuronalen Netzen können hervorragende Ergebnisse z.B. im Rahmen der Innenraumluftqualitätsmessung (IAQ) erzielt werden $[2,3]$.

Für komplexere Messaufgaben können insbesondere Informationen orthogonal zum TCO interessant sein, um eine selektivere und stabilere Modellbildung mit Methoden des maschinellen Lernens zu ermöglichen. Dies kann beispielsweise durch Kombination verschiedener MOS-Sensorschichten oder verschiedener Sensorprinzipien erfolgen. Darüber hinaus ist die Kombination mit einem Präkonzentrator denkbar, der zusätzlich Informationen über Flüchtigkeit und Diffusionsgeschwindigkeit bereitstellt [4].

Das vorliegende Manuskript gibt einen kurzen Einblick in diese Technologien. Ausgehend von der Anwendung IAQ und einer schlichten Kalibrierung eines Sensorsystems basierend auf einem einzigen MOS-Sensor, wird der Informationsgehalt der Desorption eines Präkonzentrators demonstriert sowie gezeigt, dass die Kombination von MOS-
Sensorschichten mehr Information bieten kann, aber nicht muss. Zuletzt wird gezeigt, dass im Außenbereich durch die Kombination mehrerer Sensortechnologien wie MOS-Sensoren und elektrochemische Zellen (EC-Zellen) z.B. Maskierungseffekte aufgedeckt werden können, aber auch neue Fragen aufgrund von Korrelationen zum Vorschein kommen.

\section{Methoden und Materialien}

Die vorgestellten Ergebnisse wurden mit unterschiedlichen Messsystemen und Gasmischsystemen erhalten.

Die Ergebnisse im Unterkapitel Kalibrierung beruhen auf dem Produktionsprozess eines IAQMesssystems basierend auf einem MOS-Sensor ${ }^{1}$. Die Kalibrierung im Rahmen der Produktion erfolgt innerhalb einer Gasmischanlage mit Feuchteerzeugung bei Raumtemperatur, einem Nullluftgenerator, Kohlenstoffmonoxid und Wasserstoff als Hintergrundgase, sowie der Zumischung repräsentativer flüchtiger organischer Verbindungen (VOCs).

Die Daten aus dem Unterkapitel Präkonzentrator wurden mit einem eigens hergestellten PCSM (preconcentrator sensor module) in einer Gasmischanlage vermessen, das einen Präkonzentrator mit einem MOS-Sensor in einem Keramikgehäuse vereint. Die verwendete Gasmischanlage ist in [5] beschrieben.

Das Unterkapitel Kombination von MOSSensorschichten beruht auf einem öffentlich verfügbarem Datensatz [6], der in [7] näher beschrieben ist.

Das Unterkapitel Kombination von Sensortypen beruht auf Feldinstallationen des EnvironmentalCheckerOutdoor (ECO), ein gepumptes modulares Gassensorsystem. Die gezeigten Daten zu StickoxidEvents entstammen einer Installation in einem Industriepark. Die erwähnten Korrelationen (Stickoxide, Ozon und Wasserstoff) wurden im Rahmen einer verkehrsnahen Installation beobachtet.

\footnotetext{
${ }^{1}$ Es sind weitere Sensoren im System enthalten, die für die Messung der TVOC-Konzentration keine oder eine untergeordnete Rolle spielen (Temperatur und Feuchte werden als Korrekturparameter eingesetzt).
} 


\section{Ergebnisse}

\section{Kalibrierung}

Basis jeglicher Anwendung von Methoden des maschinellen Lernens ist die statistische Kalibrierung. Hierbei werden Trainingsdaten erzeugt, auf deren Basis die Modellbildung erfolgt. Am Markt erhältliche Gassensoren besitzen häufig bereits Ausgabesignale wie die Summenkonzentration der flüchtigen organischen Verbindungen (TVOC) in Innenräumen. Die Qualität dieser Signale genügt jedoch nur bedingt dem Anspruch verschiedener Anwender, weswegen sämtliche Systeme in der Produktion nachkalibriert werden.

Abb. 1 zeigt die Signalverläufe von zwölf Sensorsystemen. Die Ausgabe der MOS-Sensoren ab Werk (uncalibrated TVOC values) sind in den beiden Graphen links dargestellt. Aus der Kombination von zwei Ausgabewerten des Sensors wird mit Hilfe einer PLSR eine neue 3S-TVOC-Ausgabe (rechte Seite, calibrated TVOC values) erzeugt. Die oberen beiden Graphen zeigen denselben Abschnitt aus der Werkskalibrierung in der Gasmischanlage. Die Gasangebote sind Gemische repräsentativer VOCs bei insgesamt $0,6-3 \mathrm{mg} / \mathrm{m}^{3}$, die verschiedene Responsespektren auf den Sensoren abdecken. Zusätzlich werden die Hintergrundgase Wasserstoff und Kohlenstoffmonoxid variiert. Die unteren beiden Graphen zeigen Signale derselben Sensorsysteme im Feld im Anschluss an die Kalibrierung über mehrere Tage - der Tag-Nach-Rhythmus der Lüftung ist deutlich zu erkennen.

Im Ergebnis weisen die kalibrierten Ausgabewerte einen vielfach besseren Gleichlauf auf, wodurch Messergebnisse in verschiedenen Räumen verglichen werden können. Darüber hinaus liegen die kalibrierten Messsignale bei deutlich niedrigeren Konzentrationen, da die Response der Sensoren auf ein für die Anwendung repräsentatives Gemisch (inkl. Störgrößen) bestimmt wurde.

\section{Präkonzentrator}

Abb. 2 zeigt die relative Signaländerung einer sensitiven Schicht des MOS-Sensors im PCSM bei Angebot verschiedener Gase nach 8 Minuten Adsorption des Gases bei einer Präkonzentrator-Temperatur von $40^{\circ} \mathrm{C}$ und einer $120 \mathrm{~s}$ langen Desorption bei $200^{\circ} \mathrm{C}$. Die Temperatur der Sensorschicht beträgt $250^{\circ} \mathrm{C}$. Während der 8-minütigen Adsorptionszeit wird der Sensor zusätzlich im TCO betrieben, um den größtmöglichen Gesamtinformationsgehalt zu erhalten.

Die unterschiedliche Höhe der Desorptionspeaks wird durch die Sensitivität des Sensors und die Flüchtigkeit und damit das Adsorptionsvermögen des Präkonzentrators bestimmt. Der zeitliche Versatz des Maximums wird neben dem Zeitverhalten des Sensors durch die Desorptionstemperatur und die Diffusionsgeschwindigkeit der Gase bestimmt. Tab. 1 zeigt einen Überblick der aus dem Sensor- signal erhaltenen Parameter des Peaks (Zeitpunkt des Maximums und relative Höhe) sowie einen Vergleich mit charakteristischen Eigenschaften der vermessenen Substanzen (Dampfdruck, Siedepunkt und Diffusionskoeffizient).

Abb. 2: Relative Änderung des Sensorleitwerts $\left(\mathrm{T}=250{ }^{\circ} \mathrm{C},\left(\left(\mathrm{G}_{\text {Desorption }} / \mathrm{G}_{\text {Adsorption }}\right)-1\right) \cdot 100\right)$ eines MOS-Sensors zu Beginn der Desorptionsphase des $\mu$ Präkonzentrators nach 8-minütiger Adsorption verschiedener Gase.

\section{Kombination von MOS-Sensorschichten}

Der zusätzliche Informationsgehalt durch die Verwendung mehrerer verschiedener MOSSensorschichten wird beispielhaft an einem online verfügbaren Datensatz demonstriert [6]. Alle Details der Messung sind in der zugehörigen Veröffentlichung von Baur et. al. beschrieben [7].

Auf Basis des temperaturzyklischen Betriebs (Zykluslänge $120 \mathrm{~s}$ ) werden für die fünf verwendeten Sensorschichten (AS-MLV-P2 und jede der vier SGP30 Sensorschichten) jeweils 240 Merkmale berechnet (Mittelwert und Steigung je $1 \mathrm{~s}$-Abschnitt). Mit diesen Merkmalen werden PLSR-Modelle trainiert und ein RMSE auf Basis einer 10-fachen Kreuzvalidierung gebildet. Diese RMSE-Werte sind als Säulendiagramm für die verschiedenen Schichten und Gase in Abb. 3 dargestellt. Zusätzlich ist der RMSE erhalten durch die Verwendung aller vier Sensorschichten des SGP30 zum Vergleich dargestellt.

Im Ergebnis wird ersichtlich, dass je nach Zielparameter, der RMSE aus der Kombination aller vier Schichten lediglich das beste Ergebnis einer der Schichten repräsentiert (Kohlenstoffmonoxid) - die Verwendung mehrerer Schichten geht also in diesem Fall mit keiner Verbesserung im Gesamtergebnis einher. Im Gegensatz dazu zeigt das Modell zur Vorhersage der Gesamt-VOC-Konzentration einen um den Faktor 2 niedrigeren RMSE mit der Kombination allen vier Schichten im Vergleich zu den einzelnen Schichten. 


\section{Kombination von Sensortypen}

Abb. 4 zeigt normierte Signalverläufe einer ECZelle für $\mathrm{NO}_{2}$ und mehrerer MOS-Sensoren im TCO im Feld. Vor allem bei Außenluftmessungen können oxidierende Gase wie Stickoxide und Ozon in Kombination mit reduzierenden Zielgasen zu Maskierung und gegenläufigen Signalen führen. In den gezeigten Ausschnitten in Abb. 4 ist das in verschiedenen Ausprägungen beispielhaft gezeigt. In einem ähnlichen Aufbau neben einer Referenzmessstation können Korrelationen zwischen den Referenzdaten für Stickoxide, Ozon und das Sensorsignal eines wasserstoffselektiven MOS-Sensors beobachtet werden. Die Variation von Wasserstoff im Außen- und Innenbereich, ist aufgrund fehlender analytischer Methoden und Interessen wenig erforscht [8]. Ob die Korrelationen eine Querempfindlichkeit des Sensors oder eine tatsächliche Korrelation der Wasserstoffkonzentration mit Stickoxiden oder Ozon darstellen, ist Gegenstand weiterer Untersuchungen.

\section{Diskussion, Fazit und Ausblick}

Die beschriebenen Ergebnisse zeigen Ausschnitte einzelner Bausteine der Sensorik und Datenauswertung komplexer Gesamtsysteme. Zahlreiche weitere Untersuchungen - intern und extern - formen das Gesamtbild und tragen so zur Entwicklung von besonders leistungsfähigen Gassensorsystemen bei.

Erwähnt seien zum Beispiel die Messung von Methan in harschen Umgebungen mit Hilfe von vier verschiedenen Sensorprinzipien [9] oder Felduntersuchungen zu MOS-Sensoren [2,8], wobei unter anderem die Blindheit des SGP30 gegenüber Limonen auffiel sowie eine Querempfindlichkeit von wasserstoffselektiven Sensoren gegenüber diesem Gas. Solche Informationen können je nach Anwendung eine wertvolle weitere Dimension darstellen und damit die Sensorauswahl beeinflussen. Eine gezielte Vorverarbeitung der zum Training verwendeten Merkmale kann wahlweise das nicht-lineare Sensorverhalten auf eine lineare Auswertemethode wie die PLSR [10] oder das Sensorverhalten auf eine nichtlineare Ausgabegröße wie die Geruchsintensität anpassen. Auf dieser Basis werden für die jeweilige Messaufgabe Werkskalibrierungen zur Verrechnung und Korrektur der Signale durchgeführt.

Bei verteilten Sensornetzwerken und der Verwendung von Metadaten wie Windrichtung wird die Gesamtbewertung der Daten in einer weiteren „Dimension“ komplexer. Basis für solche Ansätze muss stets eine bedachte Auswahl der Sensorik sowie eine gute Kenntnis ihrer Eigenschaften sein. In Kombination mit Ausbreitungsrechnungen werden diese Sensornetzwerke unter anderem zur Analyse der Geruchsausbreitung oder zur Überwachung von Industrieanlagen verwendet. Zur erfolgreichen Erschließung solcher Anwendungen müssen als Basis für die komplexen Modelle des maschinellen Lernens alle Sensorsignale ausreichend verstanden und optimiert sein, um eine Fehlklassifizierung oder -quantifizierung in verschiedenen Situationen zu vermeiden.

\section{Literatur}

[1] https://www.3s-ing.de/technologie/, aufgerufen am 12.11.2021.

[2] Baur, T. Amann, J., Schultealbert, C., Schütze, A.: Field Study of Metal Oxide Semiconductor Gas Sensors in Temperature Cycled Operation for Selective VOC Monitoring in Indoor Air, Atmosphere. 2021; 12(5):647. https://doi.org/10.3390/atmos12050647

[3] Yannick, R., Amann, J., Baur, T., Goodarzi, P., Schultealbert, C., Schneider, T. Schütze, A.: HighPerformance VOC Quantification for IAQ Monitoring Using Advanced Sensor Systems and Deep Learning, Atmosphere 2021, 12(11), 1487; https://doi.org/10.3390/atmos12111487

[4] Leidinger, M., Rieger, M., Sauerwald, T., Alépée, C., Schütze, A.: Integrated pre-concentrator gas sensor microsystem for ppb level benzene detection, Sensors and Actuators B. 2016; 236. https://doi.org/10.1016/j.snb.2016.04.064

[5] Leidinger, M., Schultealbert, C., Neu, J., Schütze, A., Sauerwald, T.: Characterization and calibration of gas sensor systems at ppb level-a versatile test gas generation system, Meas. Sci. Technol. 29 015901, 2018. https://doi.org/10.1088/1361-6501/aa91da

[6] Baur, T., Bastuck, M., Schultealbert, C.: Random gas mixtures for efficient gas sensor calibration: Dataset, 2020. https://doi.org/10.5281/zenodo.4264224

[7] Baur, T., Bastuck, M., Schultealbert, C., Sauerwald, T., and Schütze, A.: Random gas mixtures for efficient gas sensor calibration, J. Sens. Sens. Syst., 9, 411-424, 2020. https://doi.org/10.5194/jsss-9-411-2020

[8] Schultealbert, C., Amann, J., Baur, T., Schütze, A.: Measuring Hydrogen in Indoor Air with a Selective Metal Oxide Semiconductor Sensor, Atmosphere 2021, 12(3), 366. https://doi.org/10.3390/atmos12030366

[9] Lensch, H., Sauerwald, T., Müller, M., Braunecker, F., Dudziak, T., Brünnet, H., Schütze, A. : Multi-sensor system for selective methane measurements in harsh environments, SMSI 2020 - Sensors and Instrumentation, https://doi.org/10.5162/SMSI2020/P1.1

[10]Bastuck, M., Leidinger, M., Sauerwald, T., Schütze, A.: Improved quantification of naphthalene using nonlinear Partial Least Squares Regression. ISOEN 2015, 16th International Symposium on Olfaction and Electronic Noses, Dijon, France. https://arxiv.org/abs/1507.05834

[11]Jessel, W.: Gase-Dämpfe-Gasmesstechnik, Ein Kompendium für die Praxis. Dräger AG, Lübeck, 2001.

[12]GESTIS-Stoffdatenbank, https://gestis.dguv.de/, aufgerufen am 12.11.2021.

\section{Danksagung}

Unser Dank gilt dem Lehrstuhl für Messtechnik von Prof. Schütze der Universität des Saarlandes, in dessen Labor die Daten des Unterkapitels Präkonzentrator aufgenommen wurden. 

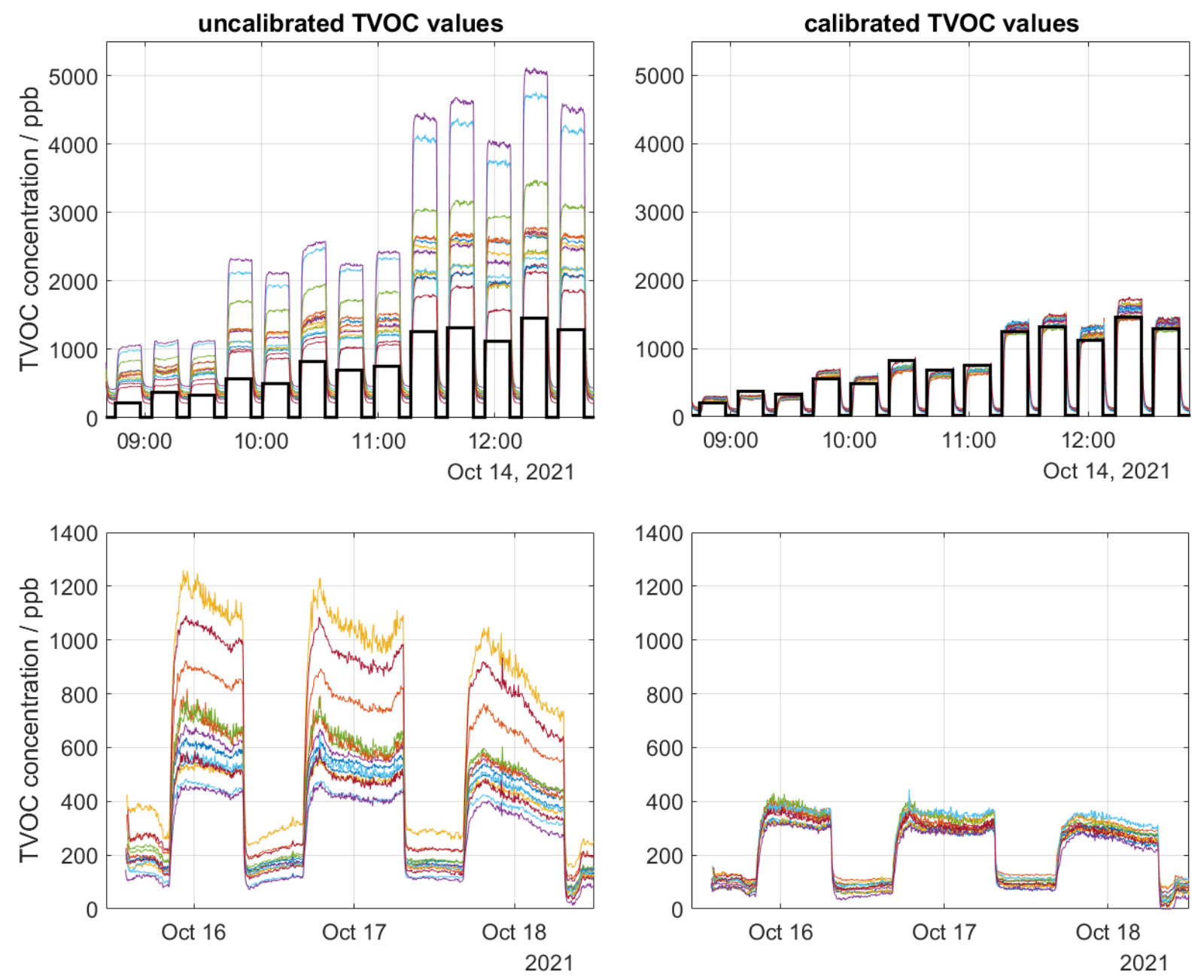

Abb. 1: Vergleich der ab Werk vom Sensor ausgegebenen TVOC-Werte (links) in der Kalibrierung (oben) mit den kalibrierten Werten erhalten durch eine PLSR-Bildung mehrerer Sensormerkmale (rechts oben). Die unteren Graphen zeigen den Vergleich beider Signale über mehrere Tage in der Produktionshalle.

Tab. 1: Desorptionszeitpunkt und Höhe im Sensorsignal des Halbleitergassensors für verschiedene Gase nach 10-minütiger Adsorption im Vergleich mit den relevanten Stoffeigenschaften Dampfdruck, Siedepunkt und Diffusionskoeffizient für die Entstehung dieser unterschiedlichen Desorptionsverhalten. (Daten aus [11,12])

\begin{tabular}{|c|c|c|c|c|c|}
\hline Gas & $\begin{array}{l}\text { Zeitpunkt des } \\
\text { Maximums in s }\end{array}$ & $\begin{array}{l}\text { Höhe Maxi- } \\
\text { mum in \% }\end{array}$ & $\begin{array}{l}\text { Dampfdruck in } \\
\text { hPa bei } 20^{\circ} \mathrm{C}\end{array}$ & $\begin{array}{c}\text { Siedepunkt in } \\
{ }^{\circ} \mathrm{C}\end{array}$ & $\begin{array}{c}\text { Diffusionskoeffizient } \\
\text { (Luft, } 20^{\circ} \mathrm{C}, \\
1013 \mathrm{hPa} \text { ) in } \mathrm{cm}^{2} / \mathrm{s} \\
\end{array}$ \\
\hline Toluol & 11,7 & 7,12 & 29,1 & 111 & 0,0763 \\
\hline Aceton & 7,6 & 16,7 & 246 & 56 & 0,1049 \\
\hline Ethanol & 9,9 & 6,68 & 58 & 78 & 0,1181 \\
\hline Formaldehyd & 8,3 & 3,99 & 4300 & -19 & \\
\hline Acetaldehyd & - & - & 1006 & 20 & \\
\hline Xylol $(\mathrm{m}, \mathrm{p})$ & 8,7 & 8,64 & 8 & 138 & \\
\hline Benzol & 13,3 & 3,76 & 100 & 80 & 0,0859 \\
\hline Wasserstoff & 6,7 & 4,80 & n.a. & $-252,9$ & 0,69 \\
\hline Kohlenstoffmonoxid & 7,9 & 1,57 & n.a. & $-191,5$ & \\
\hline
\end{tabular}




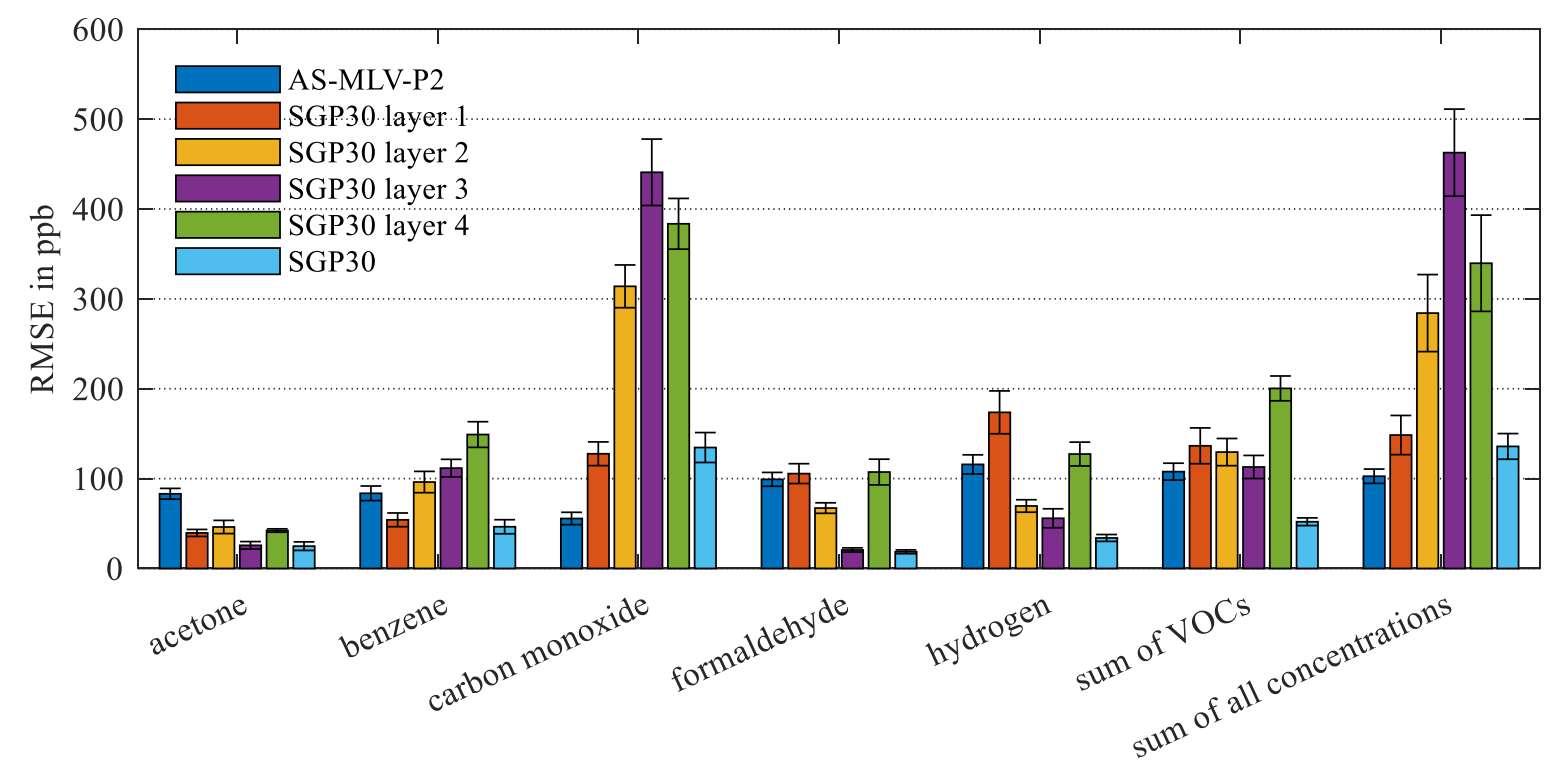

Abb. 3: Vergleich der Fehler erhalten aus der Kreuzvalidierung des Trainings auf Basis verschiedener MOSSensorschichten.

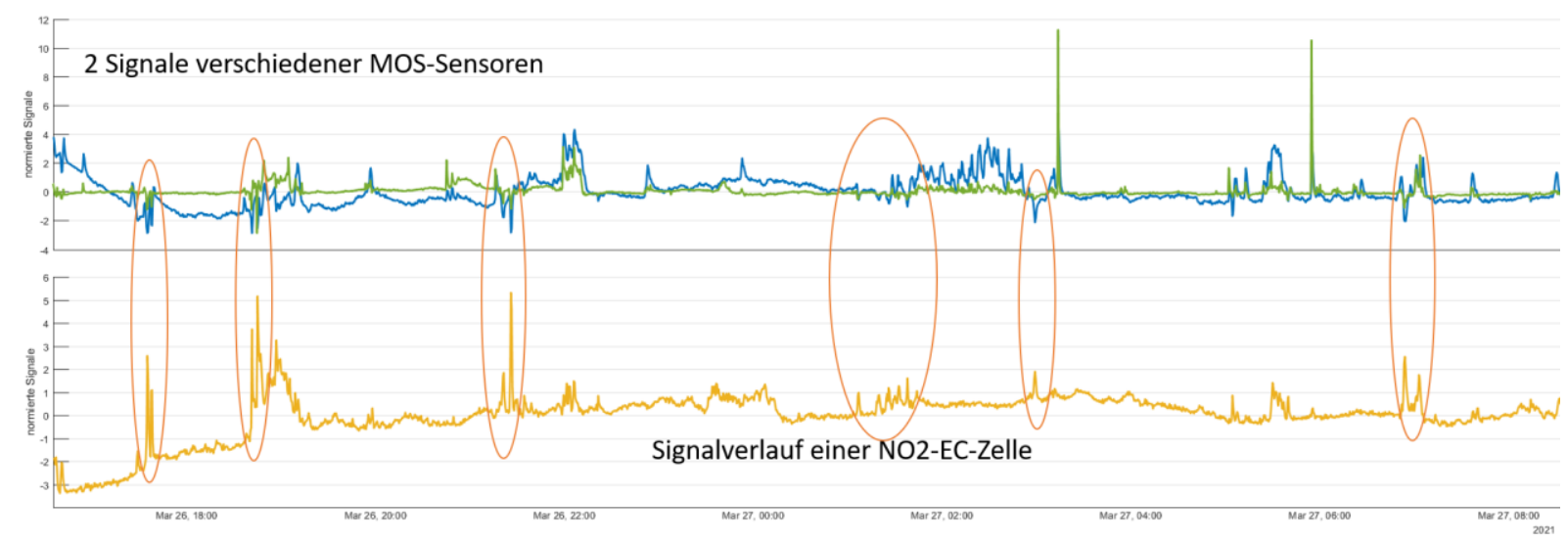

Abb. 4: Ausgewählte Events einer Messstation im Außenbereich eines Chemieparks und Vergleich der normierten Sensorsignale verschiedener Messtypen (oben: MOS-Sensoren, unten: $\mathrm{NO}_{2}$-EC-Zelle). 